\title{
Main-Chain Flexibility and Side-Chain Crystallization of Widely Spaced Comb-Like Polymers
}

\author{
Tadamichi HIRABAYASHI, Tomoko KIKUTA, Kazuo KASABOU, \\ and Kenji YOKOTA
}

Department of Materials Science and Engineering, Nagoya Institute of Technology, Gokiso-cho, Showa-ku, Nagoya 466, Japan

(Received March 16, 1988)

\begin{abstract}
Widely spaced comb-like polymers in which teeth (alkyl side-chains) appear on every four main-chain carbon atoms were synthesized as copolymers of styrene, or $\alpha$-methylstyrene with alkyl acrylates, alkyl methacrylates, or dialkyl itaconates. Alkyl groups were dodecyl, tetradecyl, hexadecyl, and octadecyl. The long alkyl side-chains of these comb-like polymers crystallized as in conventional comb-like polymers but the extent of crystallization varied with cumulative substitution of phenyl and methyl groups on the copolymer main-chains. This is due to the influence of these substituents on the flexibility of the main-chain.
\end{abstract}

KEY WORDS Comb-Like Polymer / Widely Spaced Comb-Like Polymer / Styrene / $\alpha$-Methylstyrene / Alkyl Acrylate / Alkyl Methacrylate / Dialkyl Itaconate / Alternating Copolymer / Crystallization /

In previous papers, ${ }^{1-3}$ we observed that long alkyl side-chains in several widely spaced comb-like polymers crystallize as in conventional comb-like polymers. The extent of crystallization and crystalline structure are similar both in widely spaced and in conventional comb-like polymers. A possible crystallized

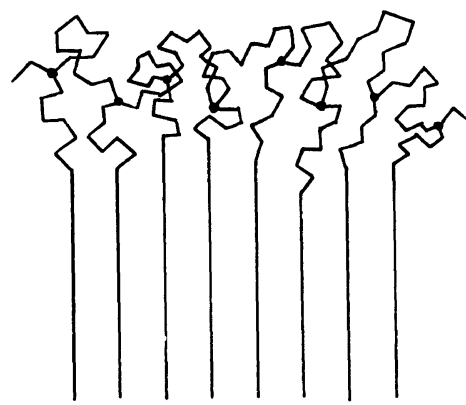

Figure 1. A proposed model for widely spaced comblike polymers. ${ }^{3}$ Parallel straight lines illustrate crystallized parts of alkyl side-chains. The problems ${ }^{11}$ of oneor two-layer packing and intra- or intermolecular crystallization are not considered here. polymer sturcture as shown in Figure 1 was proposed which contains a crystallizing part of alkyl side-chains and a noncrystallizing, random-coiling main-chain together with the rest of the alkyl side-chains. Flexible mainchain can be coiled so as to allow the sidechains to be packed in the crystal lattice. A less flexible main-chain of poly(2-hexadecyltrimethylene terephthalate) resulted in less crystallization than flexible aliphatic polyesters.

The present paper describes the synthesis, identification, and side-chain crystallization of various alternating copolymers of styrene or $\alpha$-methylstyrene with alkyl acrylate, alkyl methacrylates, or dialkyl itaconate whose alkyl groups are dodecyl, tetradecyl, hexadecyl, and octadecyl. These copolymers are considered widely spaced comb-like polymers whose teeth (the alkyl side-chains) appear on every four main-chain carbon atoms. Thus, the teeth were expected and actually found to crystallize 
similarly as those in conventional and widely spaced comb-like polymers previously studied. The extent of crystallization, however, varies with methyl substitutions on the sytrene and acrylic monomer units. This can be explained by the cumulative substituent effect of phenyl and methyl groups on the flexibility of copolymer main-chains.

\section{EXPERIMENTAL}

\section{Monomers}

Styrene and $\alpha$-methylstyrene were commercially available extra-pure reagents and purified as usual. ${ }^{4}$ Alkyl methacrylates were prepared, identified and purified as described in the previous paper. ${ }^{1}$ Ester-exchange reactions of methyl methacrylate with fatty alcohols were carried out to prepare the alkyl methacrylates. Four fatty alcohols, i.e., dodecyl, tetradecyl, hexadecyl, and octadecyl alcohols, were commercially available extra-pure reagents. The same procedures were applied to the alkyl acrylates. The dialkyl itaconates are described in a previous paper. ${ }^{5}$

\section{Polymerization}

A pair of styrene and acrylic ester monomers was placed in a nitrogen-purged glass ampoule. Ethylaluminum sesquichloride or diethylaluminum chloride $(20 \mathrm{~mol} \%$ reagent to the acrylic monomer) in toluene solution was added. Toluene solvent was added for some experiments to carry out the polymerization homogeneously. Polymerization was conducted at $0^{\circ} \mathrm{C}$ or room temperature. After a given time, the viscous or solidified polymerization mixture was poured into a large excess of methanol containing $1 \mathrm{vol} \%$ hydrochloric acid. The precipitated copolymer was collected and purified by repeated reprecipitations. Methanol or acetone was properly used as the non-solvent. For some octadecyl copolymers, hot ethanol extraction ${ }^{5}$ was necessary to remove unreacted long alkyl ester monomers.

\section{Measurements}

Copolymer molecular weights were determined by gel-permeation chromatography (GPC) on a Toyo-Soda HLC-803D with GMH, G1000H, G2000H, and G4000H TSK gel-columns and a differential refractometric detector. Tetrahydrofuran was used as the elution solvent at a flow rate of $1 \mathrm{ml} \mathrm{min}{ }^{-1}$. Copolymer molecular weights were calibrated by reference to monodisperse polystyrene standards. Nuclear magnetic resonance (NMR) spectroscopy was determined in deuterochloroform solutions on a Varian XL-200 spectrometer operated at $200 \mathrm{MHz}$ for ${ }^{1} \mathrm{H}$ or 50 $\mathrm{MHz}$ for ${ }^{13} \mathrm{C}$ nuclei. Hexamethyldisiloxane (HMDS) was used as the internal standard. Infrared (IR) spectroscopy was determined on a Nihon-Bunko IR-E spectrometer. Differential scanning calorimetry (DSC) was determined from -100 to $+150^{\circ} \mathrm{C}$ on a RigakuDenki Thermoflex with a low temperature unit. The heating and cooling rates were both $10^{\circ} \mathrm{C} \mathrm{min}^{-1}$.

\section{RESULTS AND DISCUSSION}

\section{Identification of Copolymer Structure}

The molecular weights of copolymer samples varied from 40 to 150 thousands with the copolymerization conditions such as combination of monomers, use of solvent, or copolymerization time and temperature. However, DSC experiments gave the same results for samples of a given copolymer type in this molecular weight range. Their ${ }^{1} \mathrm{H}-\mathrm{NMR}$ peak areas showed a $1: 1$ composition of styrene and acrylic units. Copolymerizations of various pairs of styrene-acrylic esters, i.e., styrene-methyl acrylate, styrene-methyl methacrylate, $\alpha$-methylstyrene-methyl acrylate, and $\alpha$-methylstyrene-methyl methacrylate, in the presence of alkylaluminum halide have been well established to give alternating copolymers. ${ }^{6,7}$ Styrene and methyl benzyl itaconate gave an alternating copolymer using diethylaluminum chloride. ${ }^{8}$ Since, under such 
copolymerization conditions, some acrylate and methacrylate monomers having various ester groups other than methyl group give alternating copolymers, ${ }^{7,8}$ the present monomers, i.e., acrylate, methacrylate, and itaconate having long alkyl groups, should certainly be expected to give alternating copolymers.

Other evidence for the alternating structures of styrene-alkyl acrylate and styrene-alkyl methacrylate copolymers was given by their ${ }^{13} \mathrm{C}$ NMR spectra. Hirai and his coworkers ${ }^{9,10}$ compared the ${ }^{13} \mathrm{C}$ NMR spectra of random and alternating styrene-methyl acrylate and styrene-methyl methacrylate copolymers and pointed out that some peaks are useful for judging sequential characteristics. Figures $2 \mathrm{a}$ and $2 \mathrm{~b}$ show ${ }^{13} \mathrm{C}$ NMR spectra of styreneoctadecyl acrylate and styrene-octadecyl

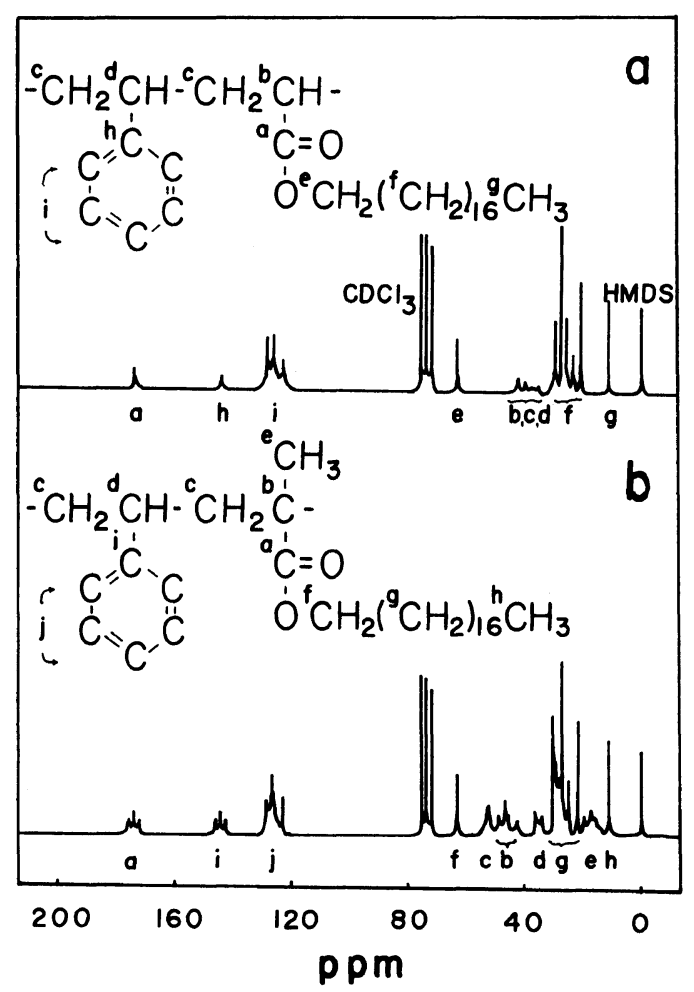

Figure 2. ${ }^{13} \mathrm{C}$ NMR spectra of alternating a) styreneoctadecyl acrylate and b) styrene-octadecyl methacrylate copolymers. methacrylate copolymers, respectively. The peak assignment indicated were carried out by referring to Hirai's spectra and INEPT (insensitive nuclear enhancement through polarization transfer) spectra. Following the observations of Hirai and others on methyl ester copolymers, the singlet $\mathrm{C}=\mathrm{O}$ peak of styreneoctadecyl acrylate copolymer in Figure $2 \mathrm{a}$ and the three split $\mathrm{C}=\mathrm{O}$, phenyl $\mathrm{C}_{1}$, and quarternary $\mathrm{C}$ peaks of styrene-octadecyl methacrylate copolymer in Figure $2 \mathrm{~b}$ are considered to demonstrate the alternating structures of these copolymers. In the same way via ${ }^{13} \mathrm{C}$ NMR spectra, the $\alpha$-methylstyrene-octadecyl acrylate and $\alpha$-methylstyrene-octadecyl methacrylate copolymers were considered to be alternating. Alternating $\alpha$-methylstyrenemethyl acrylate copolymer ${ }^{6}$ and $\alpha$-methylstyrene-octadecyl acrylate copolymer in this study showed three split $\mathrm{C}=\mathrm{O}$ and phenyl $\mathrm{C}_{1}$ peaks in both cases. Alternating $\alpha$ methylstyrene-methyl methacrylate copolymer $^{6}$ and $\alpha$-methylstyrene-octadecyl methacrylate copolymer in this study showed similar split $\mathrm{C}=\mathrm{O}$ peaks. Spectra of homopolymers were also helpful for assignment.

The split $\mathrm{C}=\mathrm{O}$ peaks show the stereochemistry of all copolymers to be atactic as in the case of methyl-ester copolymers. ${ }^{6}$

\section{DSC Study}

Figure 3 shows DSC traces for the alternating styrene-octadecyl methacrylate copolymer. The copolymer sample as precipitated was first heated from $-100^{\circ} \mathrm{C}$ to $+150^{\circ} \mathrm{C}$, then cooled to $-100^{\circ} \mathrm{C}$, and again heated to $+150^{\circ} \mathrm{C}$. On the basis of extensive studies on comb-like polymers ${ }^{11,1-3}$, the sharp endothermic peaks observed on the heating traces should be ascribed to the melting of crystallized octadecyl side-chains. The sharp exothermic peak on the cooling trace should be ascribed to the crystallization of octadecyl side-chains.

Figure 4 illustrates the cooling DSC traces for all copolymer samples. Heating traces were occasionally complicated and therefore only 
the cooling traces are discussed as in previous studies. The exothermic peaks due to sidechain crystallization of styrene-alkyl acrylate copolymers become more evident from tetradecyl to octadecyl copolymers. The peak top (crystallization temperature) shifts to the higher temperature and the peak area (heat of

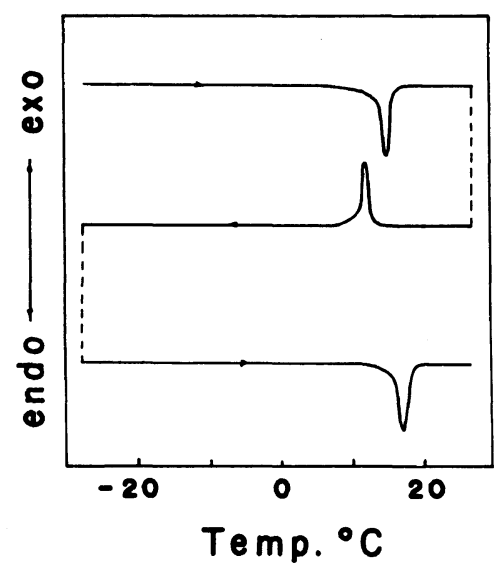

Figure 3. DSC traces for alternating styrene-octadecyl methacrylate copolymer.

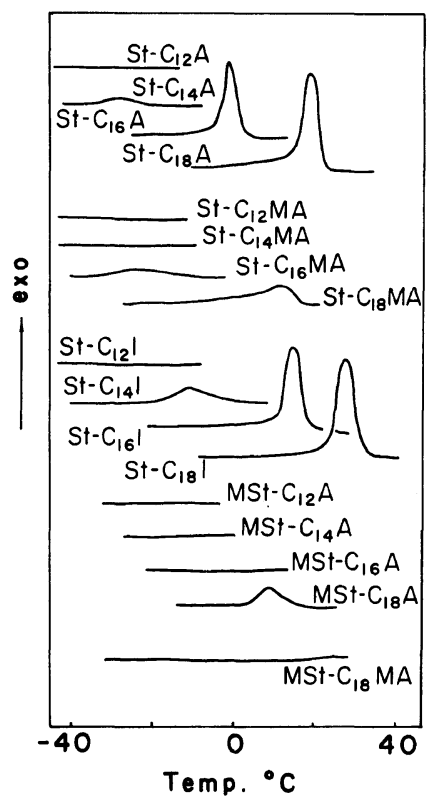

Figure 4. Cooling DSC traces for various alternating styrene-long alkyl acrylic ester copolymers. Abbreviations are given in Table $\mathrm{I}$. crystallization) increases with side-chain length. This is a general trend for comb-like polymers. ${ }^{11}$ For the dodecyl copolymer, no peak was observed under the same measurement conditions. This was also observed for styrene-dialkyl itaconate copolymers. For styrene-alkyl methacrylate copolymers, no peaks for dodecyl and tetradecyl copolymers and dull peaks for hexadecyl and octadecyl copolymers were observed. For $\alpha$-methylstyrene-alkyl acrylate copolymers, a weak peak was observed only for the octadecyl copolymer. For $\alpha$-methylstyrene-alkyl methacrylate copolymers, even the octadecyl copolymer showed no detectable peak.

DSC traces in Figure 4 are transformed into Table I which gives the crystallization temperatures $T_{\mathrm{c}}$, heats of crystallization $\Delta H_{\mathrm{c}}$, and numbers of crystallizing $\mathrm{CH}_{2}$ groups $N$. N numbers were calculated by dividing $\Delta H_{\mathrm{c}}$ by $3.08 \mathrm{~kJ} \mathrm{~mol}^{-1} \mathrm{CH}_{2}{ }^{-1}$, which is a reported value for hexagonal-packed $n$-alkanes. ${ }^{12}$ The present copolymers showed singlet IRabsorptions at $720 \mathrm{~cm}^{-1}$ which are characteristic for hexagonal-packed alkyl groups. ${ }^{10}$ For styrene-dialkyl itaconate copolymers, $N$ numbers were given for one alkyl group. Dashes

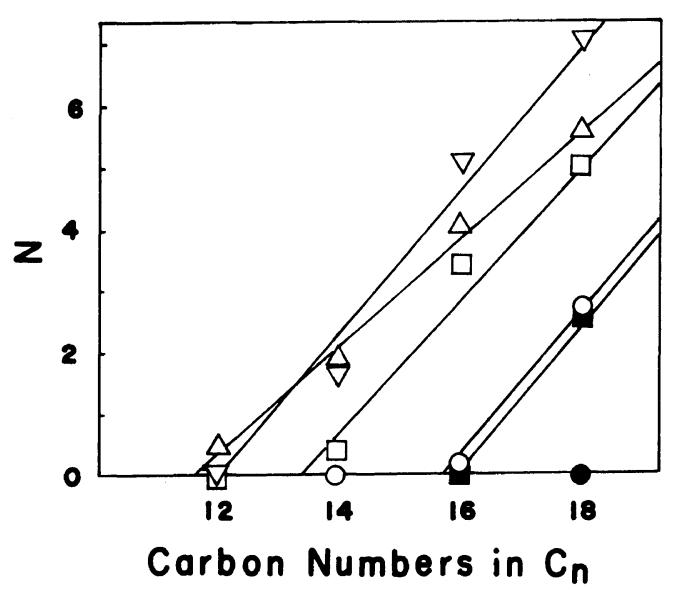

Figure 5. $N$ numbers against carbon numbers of alkyl side-chains. Copolymer types are: $\square, \mathrm{St}-\mathrm{C}_{n} \mathrm{~A} ; \mathrm{O}, \mathrm{St}-$ $\mathrm{C}_{n} \mathrm{MA} ; \nabla, \mathrm{St}-\mathrm{C}_{n} \mathrm{I} ; \square, \mathrm{MSt}-\mathrm{C}_{n} \mathrm{~A} ; \bigcirc, \mathrm{MSt}-\mathrm{C}_{n} \mathrm{MA} ; \triangle$, MA homopolymers. 
Table I. Side-chain crystallization of alternating styrene-long alkyl acrylic ester copolymers ${ }^{\mathbf{a}}$

\begin{tabular}{|c|c|c|c|}
\hline \multirow[t]{2}{*}{ Copolymer ${ }^{\mathrm{b}}$} & $\begin{array}{c}\text { Crystallizing } \\
\text { temperature } \\
T_{\mathrm{c}}\end{array}$ & $\begin{array}{c}\text { Heat of } \\
\text { crystallization } \\
\Delta H_{\mathrm{c}}\end{array}$ & $\begin{array}{c}\text { Number of } \\
\text { crystallizing } \\
\mathrm{CH}_{2}\end{array}$ \\
\hline & ${ }^{\circ} \mathrm{C}$ & $\mathrm{kJ}$ repeating unit ${ }^{-1} \mathrm{~mol}^{-1}$ & $\mathbf{N}$ \\
\hline St- $\mathrm{C}_{12} \mathrm{~A}$ & - & - & - \\
\hline$-\mathrm{C}_{14} \mathrm{~A}$ & -25 & 1.1 & 0.4 \\
\hline$-C_{16} A$ & 1 & 10.4 & 3.4 \\
\hline$-\mathrm{C}_{18} \mathrm{~A}$ & 20 & 15.5 & 5.0 \\
\hline $\mathrm{St}-\mathrm{C}_{12} \mathrm{MA}$ & - & - & - \\
\hline$-\mathrm{C}_{14} \mathrm{MA}$ & - & - & - \\
\hline$-\mathrm{C}_{16} \mathrm{MA}$ & -21 & 0.7 & 0.2 \\
\hline$-\mathrm{C}_{18} \mathrm{MA}$ & 15 & 8.4 & 2.7 \\
\hline $\mathrm{St}-\mathrm{C}_{12} \mathrm{I}$ & - & - & - \\
\hline$-\mathrm{C}_{14} \mathrm{I}$ & -12 & 9.5 & 1.5 \\
\hline$-\mathrm{C}_{16} \mathrm{I}$ & 16 & 30.5 & 5.0 \\
\hline$-\mathrm{C}_{18} \mathrm{I}$ & 29 & 42.3 & 6.9 \\
\hline $\mathrm{MSt}-\mathrm{C}_{12} \mathrm{~A}$ & - & - & - \\
\hline$-\mathrm{C}_{14} \mathrm{~A}$ & - & - & - \\
\hline$-\mathrm{C}_{16} \mathrm{~A}$ & - & - & - \\
\hline$-\mathrm{C}_{18} \mathrm{~A}$ & 9 & 8.1 & 2.6 \\
\hline $\mathrm{MSt}-\mathrm{C}_{18} \mathrm{MA}$ & - & - & - \\
\hline
\end{tabular}

${ }^{\text {a }}$ From cooling DSC traces. About $7 \mathrm{mg}$ samples were taken in an aluminum pan with a lid. $T_{\mathrm{c}}$ were the peak-top temperatures.

b Abbreviations are: $\mathrm{St}=$ styrene; $\mathrm{MSt}=\alpha$-methylstyrene; $\mathrm{A}=$ acrylate; $\mathrm{MA}=$ methacrylate; $\mathrm{I}=$ itaconate. $\mathrm{C}_{n}$ denotes carbon numbers of ester-alkyl groups.

indicate samples for which no appreciable DSC peaks were found under the measurement conditions.

The $N$ numbers in Table I are plotted in Figure 5 against the carbon numbers of the alkyl side-chains for each type of copolymer. Data $^{1}$ for polyalkyl methacrylate are plotted for comparison. Plots for styrene-dialkyl itaconate and styrene-alkyl acrylate copolymers are roughly similar with for polyalkyl methacrylates. Plots for styrene-alkyl methacrylate and $\alpha$-methylstyrene-alkyl acrylate copolymers show smaller $N$ numbers than for the preceding two copolymers. The plot for the $\alpha$ methylstyrene-octadecyl methacrylate copolymer is on the abscissa.

The results in Figure 5 can be explained on the basis of the crystallized comb-like polymer-model in Figure 1, assuming a widely spaced comb-like copolymer,<smiles>[R]OC(=O)[C]([CH])[C](C)C</smiles>

and considering cumulative substitutions at the $\alpha$ and $\gamma$ carbons by phenyl and methyl groups. Such cumulative substitutions of phenyl and methyl groups on the main-chain would make the polymer main-chain less flexible according to the extent of substitution. The less flexible main-chain would be unfavorable to the side-chain crystallization by not allowing the side-chains to be packed and would give smaller $N$ numbers. The substitution effects by phenyl and methyl groups on the polymer flexibility are easily understandable when we refer to the glass-transition temperatures of polystyrene, poly- $\alpha$-methyl- 
styrene, polymethyl methacrylate, etc. Thus, the flexibility of the present copolymers possibly be in the order:

$$
\begin{gathered}
\text { styrene-acrylate }>\text { styrene-methacrylate } \\
\approx \alpha \text {-methylstyrene-acrylate } \\
>\alpha \text {-methylstyrene-methacrylate, }
\end{gathered}
$$

which agrees with the order of $N$ numbers.

The $-\mathrm{CH}_{2} \mathrm{COOR}$ groups in styrene-dialkyl itaconate copolymers are bulky and might have decreased $N$ numbers. There are two alkyl groups, however, in one itaconate unit and hence normal $N$ numbers are observed. $N$ numbers for dialkyl itaconate homopolymers have already been reported. ${ }^{5}$ Slightly smaller $N$ numbers for styrene-acrylate copolymers than for polymethacrylate would indicate the effect of phenyl substitution.

Acknowledgement. The authors thank Iwata Chemical Industry Co., Ltd. for the research grant and gift of itaconic anhydride.

\section{REFERENCES}

1. K. Yokota, T. Kougo, and T. Hirabayashi, Polym. J., 15, 891 (1983).

2. K. Yokota and T. Hirabayashi, Polym. J., 17, 991 (1985).

3. K. Yokota and T. Hirabayashi, Polym. J., 18, 177 (1986).

4. T. Otsu and M. Kinoshita, "Kobunshi Gosei no Jikkenho," Kagaku-Dojin, Kyoto, 1972, pp 72-75.

5. T. Hirabayashi and K. Yokota, Polym. J., 19, 1115 (1987).

6. K. Yokota and T. Hirabayashi, J. Polym. Sci., Polym. Chem. Ed., 14, 57 (1976).

7. C. H. Bamford in "Alternating Copolymers," J. M. G. Cowie, Ed., Plenum Press, New York, N. Y., 1985, pp 75-152.

8. K. Yokota, unpublished data.

9. H. Koinuma, T. Tanabe, and H. Hirai, Macromolecules, 14, 883 (1981).

10. H. Hirai, H. Koinuma, T. Tanabe, K. Takeuchi, J. Polym. Sci., Polym. Chem. Ed., 17, 1339 (1979).

11. N. A. Plate and V. P. Shibaev, J. Polym. Sci., Macromol. Rev., 8, 117 (1974).

12. M. G. Broadhurst, J. Res. Natl. Bur. Stand., 66A, 241 (1962).

13. D. Chapman, J. Chem. Soc., 4489 (1957). 